\title{
Defective noncanonical autophagy in SLE-like disease
}

Defective clearance of debris has been implicated in the pathogenesis of systemic lupus erythematosus (SLE). New findings, published in Nature, now show that defects in a form of noncanonical autophagy, known as LC3-associated phagocytosis (LAP), result in defective digestion of engulfed dying cells, leading to increased inflammatory cytokine production and SLE-like disease in mice. "The demonstration that patients with SLE often have defects in the clearance of dying cells is very striking because it is extremely rare to observe an uncleared dying cell in a healthy organism," explains researcher Jennifer Martinez. "Our findings take this fundamental observation and demonstrate that the processing and degradation of dying cells is just as important as their physical uptake, and that LAP is a critical regulator of this pathway."

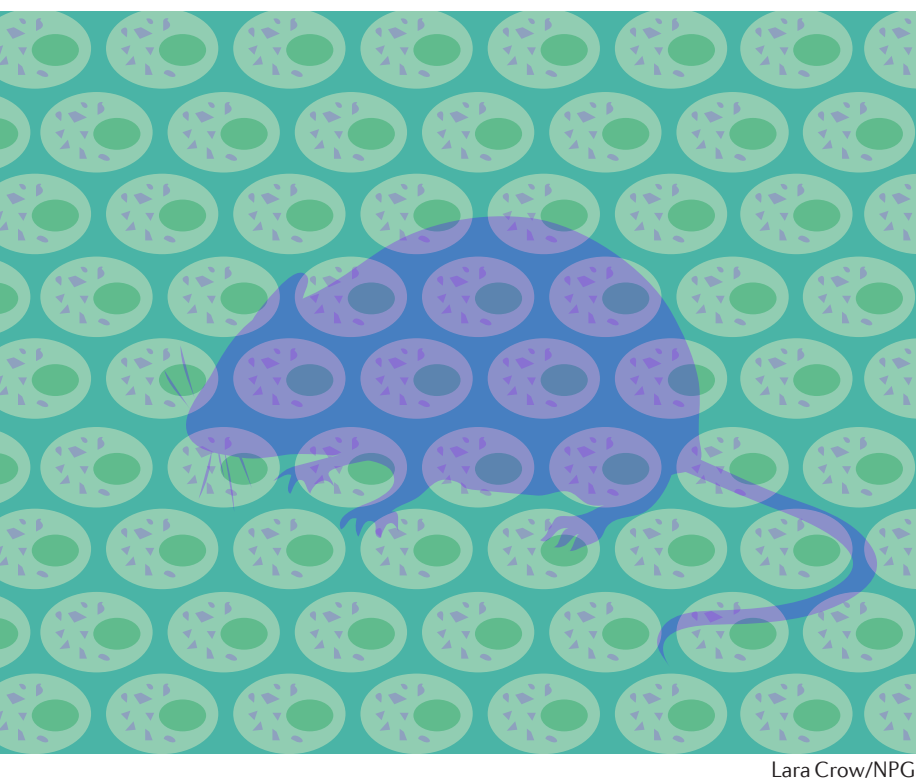

LAP is a process in which phagosomes that contain engulfed particles, such as dying cells, recruit components of the autophagy pathway to facilitate lysosomal fusion and digestion of their cargo. "We noticed that the LAP pathway was really important in establishing the correct immune response to dying cells," says Martinez. "Billions of cells die every day in a healthy adult, and it is important that the immune system tolerates this completely normal occurrence so as to avoid unwanted inflammation. Macrophages that are deficient in LAP actually generate a highly inflammatory response to dying cells, which could be very harmful and lead to autoimmunity." To investigate the effects of LAP deficiency in vivo, Martinez, Douglas Green, and colleagues generated mice with mutations in different components of the LAP and canonical autophagy pathways. "Considering the vast numbers of dying cells that an organism must silently clear over a lifetime, we allowed these mice to age," says Martinez. "Beginning at about 6 months of age, we observed that LAP-deficient mice developed a lupus-like disease, with inflammation, autoantibodies, and kidney damage."

Compared to mice with intact LAP components, LAP-deficient mice had increased inflammation, with elevated numbers of circulating lymphocytes, monocytes, neutrophils, and activated $\mathrm{CD} 8^{+} \mathrm{T}$ cells. LAP-deficient mice also exhibited increased serum levels of antidouble-stranded DNA (dsDNA) antibodies and anti-nuclear antibodies, as well as antibodies against SLE-associated autoantigens. They also showed histological and functional evidence of kidney damage, with increased serum creatinine, blood urea nitrogen, and proteinuria compared to LAP-sufficient mice, and IgG and complement C1q deposition in glomeruli, suggesting that LAP deficiency, but not autophagy deficiency, causes an autoinflammatory lupus-like syndrome in mice.

To further examine the consequences of LAP deficiency, the researchers injected dying cells into mice deficient in LAP or canonical autophagy components. Following injection, dying cells were engulfed by phagocytic cells in LAP-deficient mice but were not efficiently degraded, leading to increased production of proinflammatory cytokines. Moreover, repeated injection of dying cells into LAPdeficient mice led to the development of an SLE-like disease, with increased levels of anti-nuclear antigens and anti-dsDNA autoantibodies, and IgG and $\mathrm{C} 1 \mathrm{q}$ deposition in glomeruli.

The researchers are now planning to translate their findings from mice into humans. "We are very interested in determining if some patients with SLE have defects in LAP, what might cause such defects, and how we may be able to either re-elicit LAP or bypass the signals it generates to control SLE”, explains Green.

Susan J. Allison, Chief Editor, Nature Reviews Nephrology This article is modified from the original in Nat. Rev. Nephrol. (http://dx.doi.org/10.1038/nrneph.2016.67) 